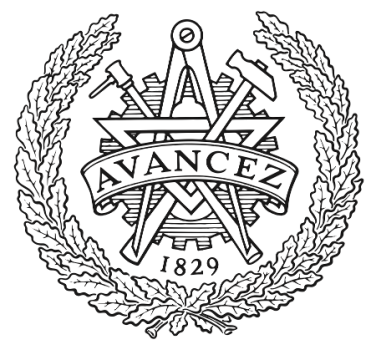

CHALMERS

UNIVERSITY OF TECHNOLOGY

\title{
Quantitative High Speed Stability Assessment of a Sports Utility Vehicle and Classification of Wind Gust Profiles
}

Downloaded from: https://research.chalmers.se, 2023-04-26 10:37 UTC

Citation for the original published paper (version of record):

Brandt, A., Sebben, S., Jacobson, B. et al (2020). Quantitative High Speed Stability Assessment of a Sports Utility Vehicle and Classification of

Wind Gust Profiles. SAE Technical Papers, 2020-April(April).

http://dx.doi.org/10.4271/2020-01-0677

N.B. When citing this work, cite the original published paper. 


\title{
Quantitative High Speed Stability Assessment of a Sports Utility Vehicle and Classification of Wind Gust Profiles
}

\author{
Author, co-author (Do NOT enter this information. It will be pulled from participant tab in \\ MyTechZone) \\ Affiliation (Do NOT enter this information. It will be pulled from participant tab in MyTechZone)
}

\begin{abstract}
The automotive trends of vehicles with lower aerodynamic drag and more powerful drivetrains have caused increasing concern regarding stability issues at high speeds, since more streamlined bodies show greater sensitivity to crosswinds. This is especially pronounced for high vehicles, such as sports utility vehicles. Besides, the competitiveness in the automotive industry requires faster development times and, thus, a need to evaluate the high speed stability performance in an early design phase, preferable using simulation tools. The usefulness of these simulation tools partly relies on realistic boundary conditions for the wind and quantitative measures for assessing stability without the subjective evaluation of experienced drivers. This study employs an on-road experimental measurements setup to define relevant wind conditions and to find an objective methodology to evaluate high speed stability. The paper focuses on the events in proximity to the drivers' subjective triggers of instability. Wind direction and magnitude, vehicle motion response, along with the subjective event triggering were measured at different conditions of the natural wind. A statistical approach was utilized to analyze the correlation between the vehicle response and subjective triggers together with the wind conditions. A correlation was established between the subjective triggers and a rapid change in lateral acceleration and yaw velocity response. The paper also proposes a set of four crosswind gust profiles of interest for driving stability, combining results from previous research and the experimental data of the natural wind obtained in this study. These findings can be used as objective measures for virtually assessing stability performance and as realistic boundary conditions for simulating wind gusts.
\end{abstract}

\section{Introduction}

The term high speed stability is often used to describe the driving stability performance of a vehicle traveling in a straight line above $100 \mathrm{~km} / \mathrm{h}$. Poor high speed stability performance will quickly fatigue the driver and reduce the vehicle's comfort level. In extreme wind conditions, poor stability performance has been shown to increase road vehicle accidents, especially for high vehicles such as trucks and vans [1]. Nevertheless, the straight line drivability may still be affected without any crosswinds [2]. High speed stability can thus be divided into crosswind sensitivity and straight line drivability, and there is no simple answer to whether these two attributes are affected by the same vehicle characteristics. The assessment of high speed stability is often done subjectively by test drivers at designated test tracks or on public roads, such as the Autobahn in Germany.

The elementary explanation of why driving stability performance deteriorates at high speeds is both that the aerodynamic forces and moments increase combined with a higher dynamic sensitivity of the vehicle. As shown in Equation 1 , the aerodynamic side force, $F_{S}$, increases exponentially with flow velocity, $V_{\mathrm{mag}}$. The density of air, $\rho$, and frontal area, $A$, can be set as the constant $K_{1}$ to simplify the expressions. The coefficient of side force, $C_{S}$, is a function of the incoming flow angle, $\psi$. So, the forces' and moments' quadratic increase with flow velocity holds for a constant flow angle. However, as the incoming flow angle decreases with increasing vehicle velocity, $v_{x}$, see Figure 1. a more realistic setting for high speed driving is to keep the side wind velocity, $w_{y}$, constant. This scenario, without head- or tail wind $\left(w_{x}=0\right)$, can demonstrate the simplified expression of the side force coefficient in Equation 2 Howell and Panigrahi [3] presented that the first approximation, in Equation 2 with the side force coefficient directly proportional to the flow angle has been seen for many vehicle models. This linearization is presented using a constant, $K_{2}$. The second approximation of small angles, together with the assumption of Reynolds number independent aerodynamic coefficients, implies that the vehicle velocity is high, e.g. above $100 \mathrm{~km} / \mathrm{h}$, which is in the range of interest for high speed stability. The resulting side force expression in Equation 3 shows an approximately linear increase with vehicle speed and side wind velocity. Hence, doubling the driving speed will double the side force, even though the side wind magnitude is kept constant. The same approximations can be done for the aerodynamic yaw moment, $M_{z}$. In summary, this simplified example shows that the increase in side force and yaw moment occur simultaneously as the yaw dampening of the vehicle is decreasing with increasing speed, making the vehicle more yaw sensitive at high speeds. These two facts exemplify why high vehicle velocity affects the stability performance of a road vehicle.

Early analytical work on driving stability concluded that the center of pressure should be located behind the neutral steering point for a vehicle to be aerodynamically stable [4, 5]. However, this is not the case for typical passenger cars, meaning that a crosswind yaws the vehicle so that the relative flow angle is increased. Stabilizing fins has been

$$
\begin{aligned}
F_{S} & =\frac{1}{2} \rho A C_{S}(\psi) V_{\text {mag }}^{2}=K_{1} C_{S}(\psi) V_{\text {mag }}^{2} \\
C_{S}(\psi) & \underset{\substack{\downarrow \\
\text { Linear }}}{\approx} K_{2} \psi=K_{2} \tan ^{-1}\left(\frac{w_{y}}{v_{x}}\right) \underset{\substack{\downarrow \\
\text { Small angles }}}{\approx} K_{2} \frac{w_{y}}{v_{x}} \\
\Rightarrow F_{S} & =K_{1} K_{2} w_{y}\left(v_{x}+\frac{w_{y}^{2}}{v_{x}}\right)
\end{aligned}
$$

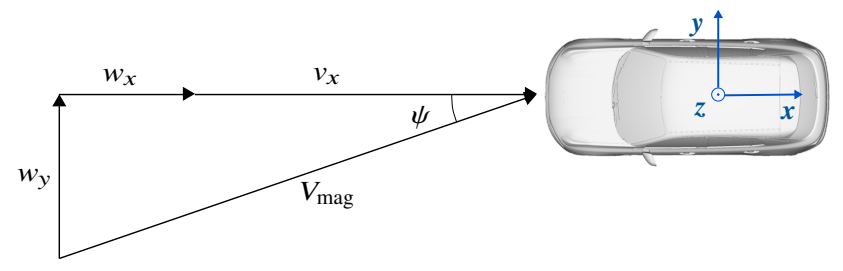

Figure 1: Schematics of how the flow angle, $\psi$, and flow velocity, $V_{\text {mag }}$, relate to the vehicle velocity, $v_{x}$, and the horizontal wind components, $w_{x}$ and $w_{y}$. 
suggested to move the center of pressure rearwards at crosswinds [6], but have not been realized due to manufacturing and aesthetic reasons. Milliken et al. [7] introduced a static stability index in 1976, based on a bicycle model with a linear tire model. The negative values of the index were defined as stable. It was shown that the value of the index increased with the vehicle velocity, agreeing with the decreased yaw dampening at higher velocities (an oversteered vehicle would reach a stability index of zero at its critical velocity). The stability index has been used in parametric studies, showing that a positive pitching aerodynamic moment increases the stability of the vehicle [8]. This correlation has also been found in subjective on-road driving studies by Howell and Le Good [9 10], indicating that the aerodynamic lift forces are important properties for the stability of the vehicle. The positive pitching is given by lower lift forces at the rear axle compared to the front. Nevertheless, the stability index is a static measure and cannot describe the dynamic on-road behavior of vehicles.

A vehicle traveling on the road is subjected to unsteady flow conditions due to the turbulence in the natural wind, flow disturbances caused by other vehicles and obstacles at the road side [11]. An important aspect of driving into crosswinds is that there is a time delay between the crosswind flow at the front and rear of the vehicle, causing overshoots in the aerodynamic yaw moment, as discussed by Chadwick et al. [12]. This was also presented by Hucho and Emmelmann [13], using slender body theory. Experimental studies have shown that the on-road turbulence affects the aerodynamics of road vehicles significantly, where recorded turbulence intensities of up to $15 \%$ were seen in freeway traffic [14-16]. A review based on these results showed that this difference compared to the low turbulence intensity of most wind tunnels could change the optimum design of, e.g., the backlight angle of a vehicle [17]. Furthermore, the driver reaction will attempt to correct for the deviations caused by the wind gusts in the unsteady flow, but it has been demonstrated that the driver response might amplify the vehicle's deviation and instability at a frequency range of $0.5-2 \mathrm{~Hz}[18]$. At frequencies $<0.5 \mathrm{~Hz}$, the driver can correct for the slow changes and at frequencies $>2 \mathrm{~Hz}$, the changes are too rapid for the driver reaction [18], and the spectral energy of the flow is also low at these frequencies [11] 19].

To enhance the reproducibility of the vehicle response to a crosswind gust, test tracks with crosswind generators have been utilized [5, 20 [22]. The International Standard ISO 12021:2010 [23] has been formulated for experiments at these facilities. The guidelines in the ISO 12021:2010 standard include a methodology where a vehicle is driven into a zone of $20 \mathrm{~m} / \mathrm{s}$ crosswind, resulting in a flow angle of $\psi=35.8 \mathrm{deg}$ at a vehicle velocity of $100 \mathrm{~km} / \mathrm{h}$. The wind profile experienced by the vehicle driving through a zone of strong crosswind has been mathematically formulated by Favre and Efraimsson [24], and is graphically presented in Figure 2 and mathematically formulated inEquation 4 This, and similar gust profiles have since been utilized in several numerical studies of crosswind sensitivity [25-28]. These extreme winds of $20 \mathrm{~m} / \mathrm{s}$ create high aerodynamic forces and a distinct motion response of the vehicle, useful for measuring differences between vehicles and configurations. However, it has also been shown that these crosswinds are too extreme to represent most real driving scenarios [14 16, 19, 29], and are more likely investigations of extreme crosswind sensitivity, rather than the actual high speed stability performance. When conducting on-road measurements of wind gust in Germany, Theissen and Wojciak [19 29] found that the typical magnitude of the crosswind generated flow angles between $2-10 \mathrm{deg}$, and that a zero-crossing of the flow angle occurred in $72 \%$ of the gusts. MacAdam et al. [5] demonstrated that the implication of a zero-crossing of the crosswind has a large influence on driving stability and subjective rating. By placing crosswind fans on alternating sides of the test track it was found that the same fan output power doubled the lateral g-force on the driver which resulted in worse subjective ratings, compared to placing the fans on one side of the test road as usually done at

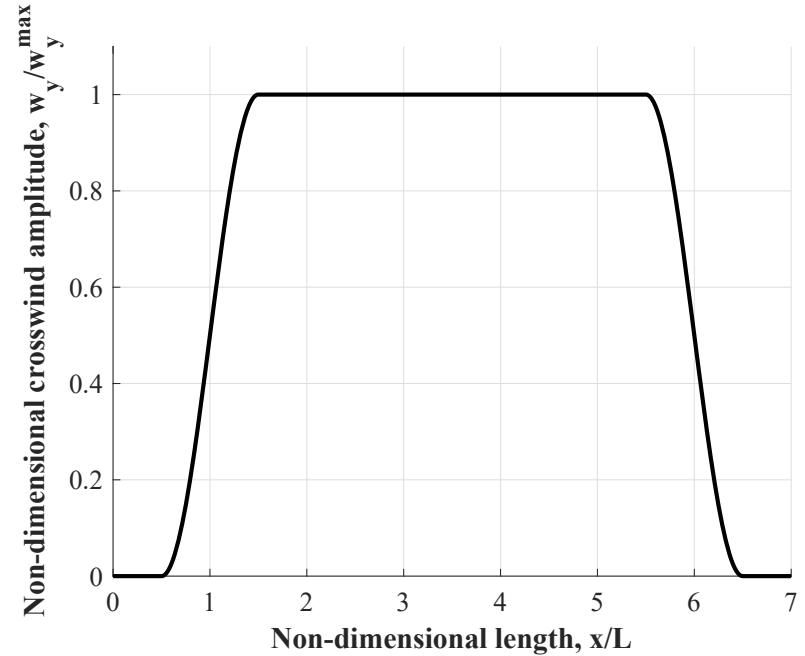

Figure 2: Crosswind profile for driving through a zone of strong crosswind, built from Equation 4 formulated by Favre and Efraimsson 24.

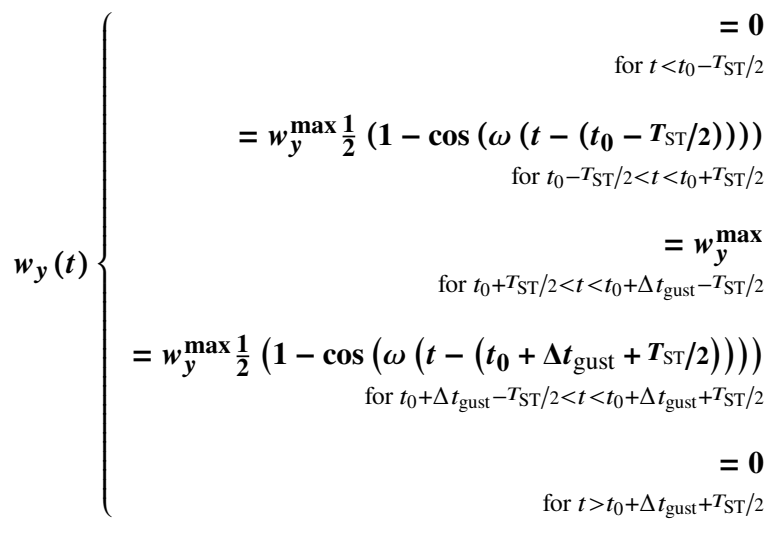

crosswind facilities. Furthermore, the final assessment of high speed stability performance is often done by experienced test drivers at test tracks. Their subjective judgment of the vehicle has proven to be reliable and reproducible. However, their subjective evaluation cannot directly be used in any virtual vehicle dynamics computer simulation and there is a need to correlate the subjective assessment to objective quantities of the vehicle motion. Some studies have found correlations between the total subjective evaluation of drivability at high speed to objective measures [2, 30]. Other studies at crosswind facilities have indicated that the vehicle motions; yaw velocity, lateral acceleration and head-rest acceleration (including roll velocity) give the best correlation to the subjective ratings [5, 31].

Although a relatively large amount of work is available in the literature, there is still need for a better understanding of how wind conditions affect high speed stability, and how to quantitatively measure the stability performance. The first goal of this experimental study focuses on correlating events of stability issues to the wind conditions, to examine the relevant loads for high speed stability. A description of the technical system is sketched in Figure 3 Furthermore, the paper expands on the knowledge of realistic crosswind gusts and proposes a mathematical formulation of these real-world inspired profiles, in similarity with the work by Favre and Efraimsson [24], to facilitate appropriate boundary conditions for flow simulations.

The second part of the paper aims to conclude the relevant vehicle motions specifically related to high speed stability, and to find quantitative objective assessment criteria for the stability performance, see 


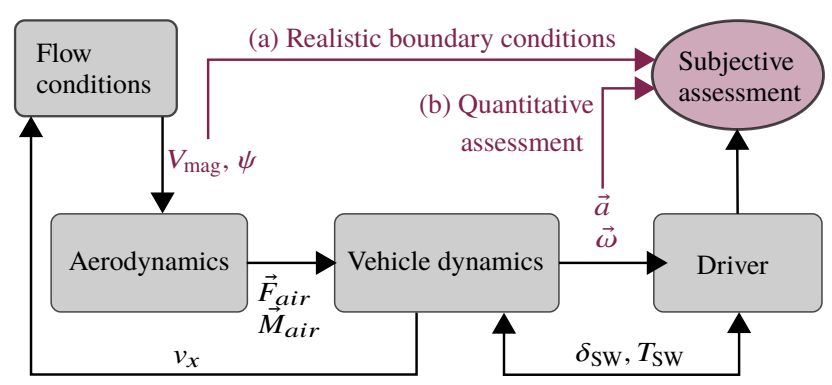

Figure 3: System description for high speed stability. The purple arrows represent the two analysis conducted in this study. The first to find realistic crosswind conditions and the second to find objective measure for high speed stability.

process (b) in Figure 3 To do so, the study analyzes the short events where experienced test drivers triggered for stability issues, to monitor the vehicle motion before each trigger event to conclude what quantitative measures that best represents the poor high speed stability performance. The two-way interaction via the steering wheel angle, $\delta_{\mathrm{SW}}$, and torque, $T_{\mathrm{SW}}$, is not assessed in this paper, since the purpose of objective quantification was to find measures independent of the driver steering response.

\section{Methodology}

This section describes the experimental setup used to conduct the test track measurements and the subsequent post-processing and analysis of the test data.

\section{Experimental Setup}

The experimental testing was conducted during six weeks with varying wind conditions. All tests were performed on dry asphalt by experienced drivers.

\section{Test Vehicle}

A compact sports utility vehicle (SUV) was used as the test vehicle in this study, see Figure 4 The vehicle was front wheel driven, had a total length of $4.51 \mathrm{~m}$, a height of $1.60 \mathrm{~m}$, a width of $1.86 \mathrm{~m}$ and a wheel base of $2.73 \mathrm{~m}$. The curb weight of the vehicle was $1856 \mathrm{~kg}$, with $56 \%$ of

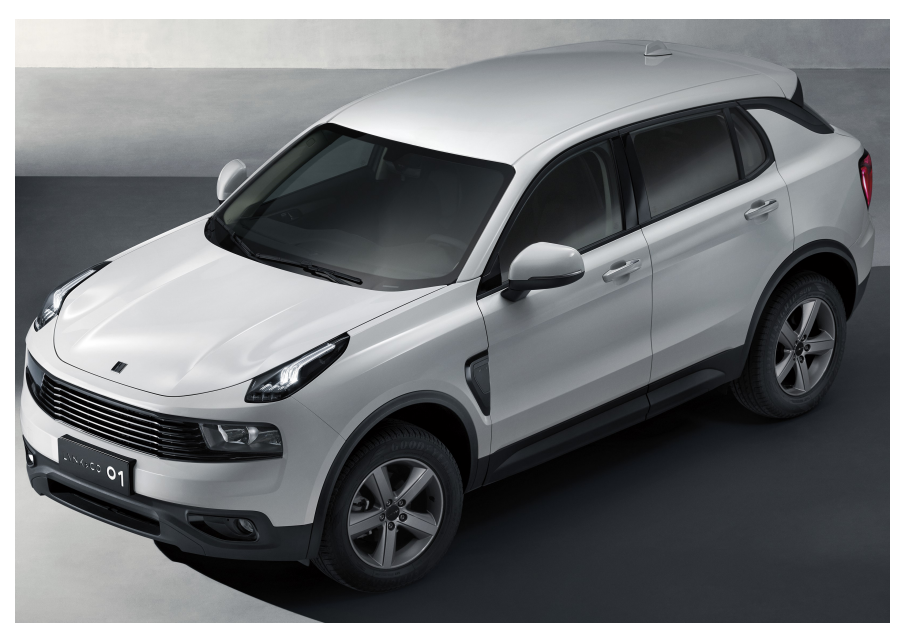

Figure 4: The vehicle model used in the experimental study.

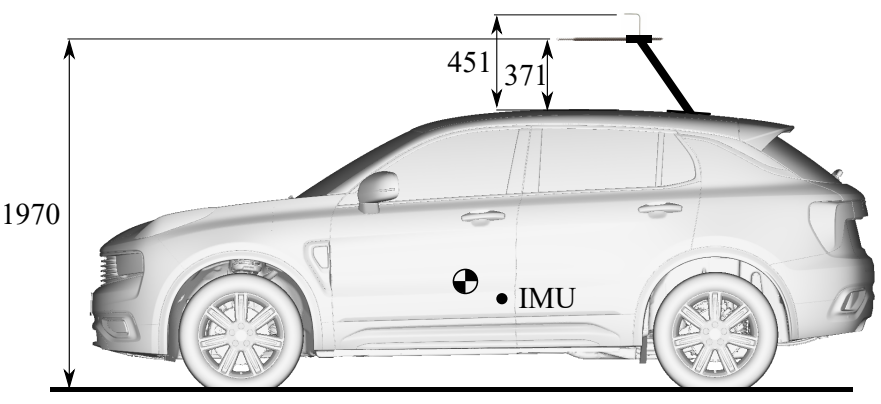

Figure 5: Schematics of the placement of the 7 hole probe, the Prandtl tube and the inertial measurement unit (IMU).

the static load on the front axle. The vehicle was fitted with new 235/50 R19 tires. The suspension system consisted of a MacPherson front suspension and a 4-link trailing arm rear suspension. Coil springs and passive dampers control the suspension system. The steering system has a steering rack with an electrical power-assisted servo function.

The test vehicle was equipped with a special rear spoiler that had proven to cause issues with high speed stability.

\section{Instrumentation}

The instrumentation setup was designed to enable measurements of the aerodynamic flow conditions together with the dynamic motion of the vehicle. All data were synchronized in a 24-bit Dewesoft Sirius HD STG-S module used as the data acquisition (DAQ) system. All data were sampled at $500 \mathrm{~Hz}$. The equipment used for the measurements is presented in Table 1

The local flow magnitude and angle subjected to the vehicle were measured using a 7 hole probe, with a conical tip of $3.18 \mathrm{~mm}$. The probe had a flow cone angle of receptivity of $70 \mathrm{deg}$ and an accuracy of $\pm 1 \mathrm{deg}$ [32]. The probe was placed $371 \mathrm{~mm}$ above the roof to measure the undisturbed flow, see Figure 5 The probe holder was mounted in place of the shark fin antenna, to minimize the effect on the flow over the rear spoiler. The probe's pressure tubes were connected to SensorTechnics HCLA0025DB pressure sensors sampling at $2500 \mathrm{~Hz}$ (although downsampled to $500 \mathrm{~Hz}$ for the final analysis). The pressure sensors measured the pressure difference between the holes at the tip of the 7 hole probe and a reference pressure. The atmospheric pressure was used as the reference pressure. This was done by mounting a Prandtl tube $80 \mathrm{~mm}$ above the 7 hole probe, see Figure 5 and using the static pressure port of the Prandtl tube as the reference pressure for the sensors. The flow magnitude, $V_{\mathrm{mag}}$, and angle, $\psi$, was calculated using the probe's calibration map with the port pressures as the input. The static port pressure of a Prandtl tube is slightly affected by yawed flow, but the pressure error was assumed to be $<2 \%$ for flow angles below $10 \mathrm{deg}$ [33], and this did not affect the flow angle calibration only the slight variation in the flow magnitude calibration.

Table 1: Instrumentation setup.

\begin{tabular}{ll}
\hline Equipment & Measurement \\
\hline -7-hole probe & \\
-Prandtl tube & Flow magnitude and angle \\
•Pressure sensors & \\
\cline { 2 - 2 } -Subjective trigger & Instability events \\
\cline { 2 - 2 } •GPS-RTK & Positioning and speed \\
\cline { 2 - 2 } •IMU & Vehicle motion response \\
\hline
\end{tabular}


To enable analyzes of the short events where particularly substantial stability problems were noted, a subjective trigger was installed in the cabin. The button on the trigger could be pressed by the driver while driving, generating a time mark in the data. The drivers were instructed to only focus on the most prominent high speed stability issues.

Two GPS antennas were mounted inside the vehicle, on the center line at the wind shield and at the rear of the vehicle. The GPS positioning was enhanced by a real-time kinematic (RTK) system, giving a positioning accuracy of $\pm 0.01 \mathrm{~m}$ and velocity accuracy of $\pm 0.1 \mathrm{~m} / \mathrm{s}$ [34].

The vehicle motions were measured using a Dewesoft DS-IMU2 module, an inertial measurement unit (IMU) that combines gyroscopes and accelerometers with measurement accuracies of $\pm 0.033 \mathrm{deg} / \mathrm{s}$ and $\pm 0.032 \mathrm{~m} / \mathrm{s}^{2}$, respectively [34]. The unit was firmly mounted to the structure of the vehicle, close to the center of gravity (CoG), see Figure 5 Exact measurements at the $\mathrm{CoG}$ could be done utilizing the IMU software to translate the acceleration values to any point in reference to the IMU. A reference point at the position of the lower back of the seated driver was used in this study to measure motions experienced by the driver.

\section{Test Track and Test Procedure}

All tests were conducted at Hällered Proving Ground, on an oval test track used for high speed testing. Three drivers participated in the study. Before the data acquisition, a co-driving session was conducted where all drivers independently could trigger at events of substantial stability issues. As all drivers marked the same events and it was concluded that the data from all three drivers could be used in the study. The data were automatically acquired, starting and stopping at the same GPS coordinates, for the two $1.1 \mathrm{~km}$ straight runs on the oval test track.

The test drivers were instructed to drive in a straight line and to keep the steering wheel fixed. Other vehicles were present on the test track, occasionally causing driver interventions or major overtaking flow disturbances. In these cases, the experiments were aborted and the data disregarded. Less obvious interference by other vehicles could not be addressed. The test procedure started with driving a couple of laps on the test track to monitor that the measurement equipment was working and that the tires had reached operational temperature. The testing was then conducted at four different velocities; $140 \mathrm{~km} / \mathrm{h}, 155 \mathrm{~km} / \mathrm{h}$, $170 \mathrm{~km} / \mathrm{h}$ and $185 \mathrm{~km} / \mathrm{h}$. Each velocity was held constant for three runs at each of the two straights, before changing velocity. To ensure significant results in an environment of low repeatability, a large data set of 407 straight line recordings $(448 \mathrm{~km})$ were collected, including 255 subjective trigger events.

\section{Post-processing}

The flow and vehicle motion data were analyzed at the subjective trigger events and compared with the complete data set to find exceptional trends in the trigger data. This section also describes the predictive classification filter that was used to find objective measures to be used in a quantitative assessment of high speed stability. All test data were filtered using a Hamming low pass filter of order 500 with a cut-off frequency of $5 \mathrm{~Hz}$. Higher frequencies of the wind and vehicle motion were disregarded for the high speed stability analysis. The longitudinal and lateral wind components, $w_{x}$ and $w_{y}$, were calculated from the flow magnitude, $V_{\mathrm{mag}}$, flow angle, $\psi$, and vehicle velocity, $v_{x}$, as in

$$
w_{x}=V_{\mathrm{mag}} \cos (\psi)-v_{x}, \quad w_{y}=V_{\mathrm{mag}} \sin (\psi) .
$$

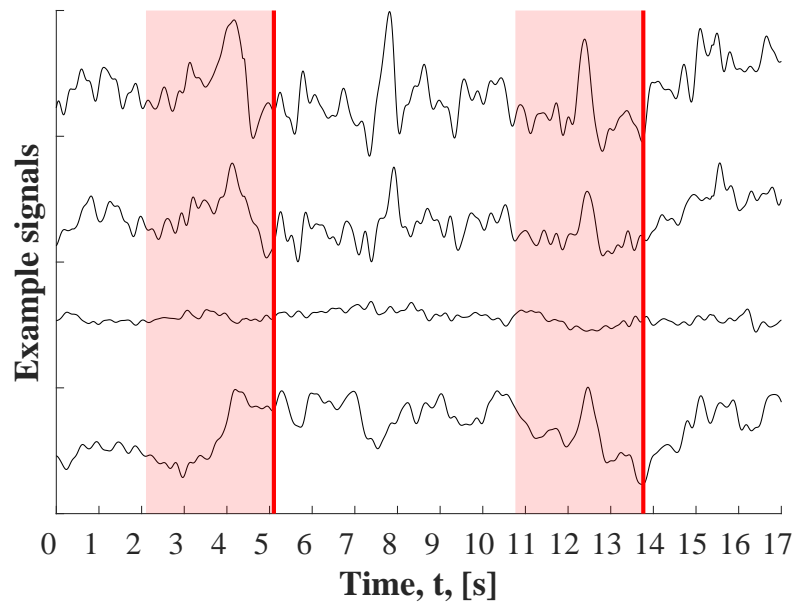

Figure 6: Example of trigger events and visualization of the regions of instability.

\section{Subjective Trigger Event Analysis}

The time marks from the drivers' subjective triggers were used to analyze the data before the instability events. Figure 6 shows examples of two trigger events as red vertical lines during a test run. A window of $3 \mathrm{~s}$ before each trigger was marked as the region of instability. It was assumed that the cause of the subjective perception of stability issues would be found within these time intervals, both in terms of the vehicle motion response and crosswind conditions.

The data before the trigger events were analyzed using various methods. The most robust and fruitful was by measuring the maximum amplitude difference between peaks within the regions of instability. This was done both for the aerodynamic flow measurements, to determine typical crosswind conditions before the subjective triggers, see (a) in Figure 3 and for the motion responses experienced by the drivers, to correlate their subjective assessment to quantitative objective measures, see (b) in Figure 3 The amplitude (peak-to-peak) values were then sorted into intervals to present the distribution of their frequency of occurrence.

\section{All Data Analysis}

The data at the subjective trigger events were compared to the complete data set, to find exceptional trends in the trigger data. The comparisons were made by using a similar analysis methodology for the complete data set. A sliding window of $3 \mathrm{~s}$, with $1 \mathrm{~s}$ stepping, was applied to all the data. The maximum amplitude difference between peaks was measured at each step. The amplitude values was then sorted into intervals to present the distribution of their frequency of occurrence, so that it could be compared to the trigger data.

\section{Predictive Classification Filter of Subjective Triggers}

To further investigate the structures in the vehicle motion data that could explain the drivers' subjective triggers, a predictive filter was designed. The filter intended to describe the filter parameters in terms of objective measures and to examine what parameters and parameter limits that could best predict the subjective trigger events, based on the objective data. When a good prediction was found, the filter parameters themselves would give information on suitable objective measures for high speed stability. 


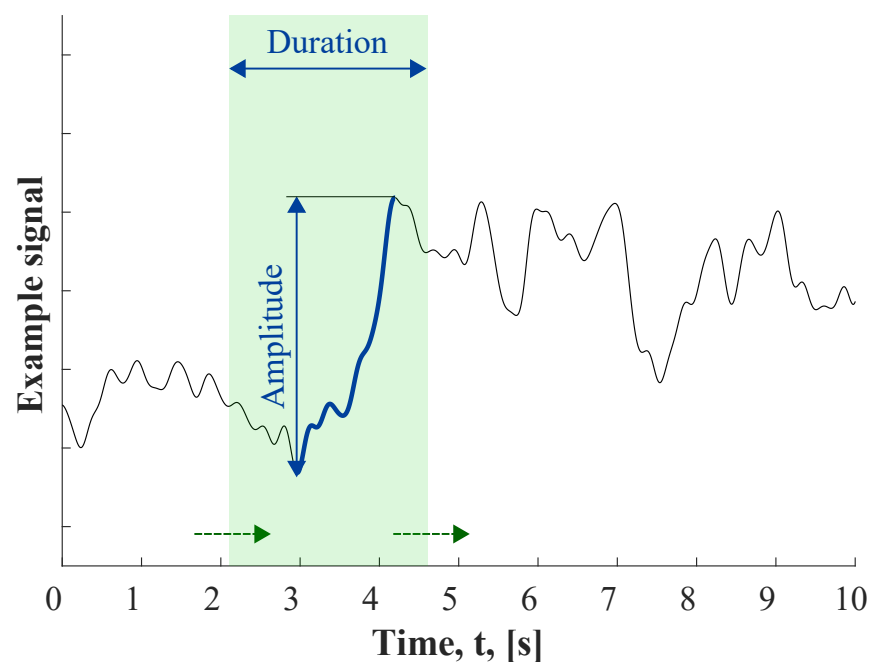

Figure 7: The moving filter, predicting event triggers based on the signal amplitude within the sliding window. The thick blue line indicates that the amplitude limit parameter of the filter was exceeded and that the change happened within the time duration limit parameter.

The prediction filter ended up with two parameters, where the first was a minimum limit of the signals' maximum amplitude within a time duration window. The duration was the second filter parameter, shown in Figure 7 If the measured amplitude within the window exceeded the amplitude limit, the data between the peaks were predicted to have stability problems (blue in Figure 7). The time duration window was sliding over the signals, measuring maximum amplitudes within the window. By decreasing the time duration, the filter would limit the vehicle motions to only predict triggers when the signals changed rapidly. It was assumed that both the amplitude and the rate of change caused a subjective assessment of poor high speed stability performance.

A set of rules was established to evaluate the accuracy of the filter predictions. If the predictions of instability occurred within the region of instability, i.e. within $3 \mathrm{~s}$ before a real event trigger, they were counted as true positive (TP) predictions. If several predictions were found within the region of instability, they would still count as one true positive. If no prediction was found within a region of instability it counted as a false negative (FN). If predictions were found outside any region of instability, they were counted as false positives (FP). Furthermore, to penalize outcomes where all data were predicted to have stability issues, any prediction longer than $4 \mathrm{~s}$ was counted as two predictions and longer than $8 \mathrm{~s}$ as three, and so on. Figure 8 shows an example of a signal including one real subjective trigger event and three predicted motions of stability issues. The first two predictions fall within the region of instability and were thus counted as one true positive. The third prediction was longer than $4 \mathrm{~s}$ and was therefore counted as two false positives. No false negative prediction occurred, where a region of instability was found without any predictions within. The confusion matrix for the example in Figure 8 can be seen in Table 2

The values in the confusion matrix were used to optimize the filter parameters. A loss function, $f_{L}$, was balanced to promote the true

Table 2: Confusion matrix for the example in Figure 8 with one true positive (TP), no false negatives (FN) and two false positives (FP). The true negative (TN) is not applicable in this method.

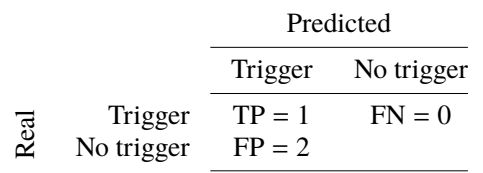

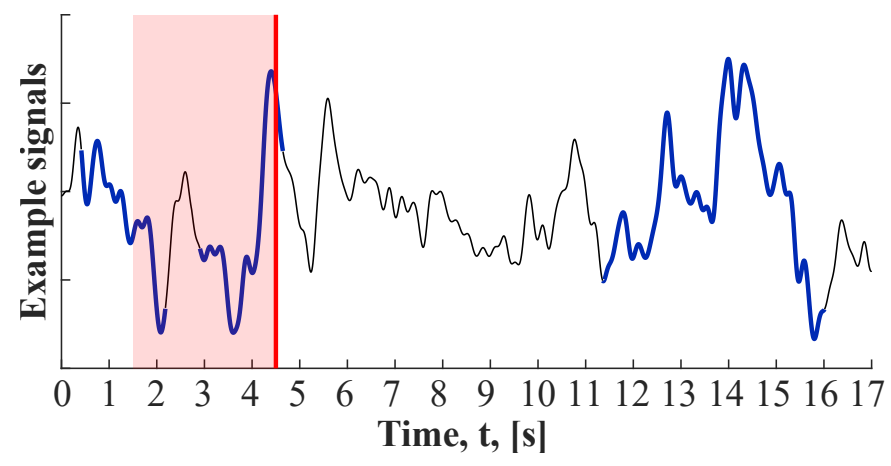

Figure 8: Example of a data recording including one subjective trigger event (red). The filter predicts three events of stability issues (blue), whereas the first two occur within the region of instability and were counted as one true positive (TP). The last predicted event was longer than $4 \mathrm{~s}$ and was thus counted as two false positives (FP)

positive triggers and to penalize the false negative and false positive triggers, as

$$
f_{L}=-1.5 \cdot \mathrm{TP}+1 \cdot \mathrm{FN}+0.25 \cdot \mathrm{FP}
$$

The loss function would take its minimum (best) value when no false triggers occured and all real triggers were predicted by the filter, in this study a value of -304.5 . An optimization strategy, using differential evolution [35], for minimizing the loss function value by varying the magnitude and duration limits of the filter was employed. The optimization methodology was conducted on the six vehicle motions; longitudinal acceleration, lateral acceleration, vertical acceleration, roll velocity, pitch velocity, and yaw velocity seperately.

\section{Results}

\section{Wind Gust Conditions}

The varying wind intensity between and throughout the days of data acquisition gave a broad perspective on the crosswind influence on high speed stability. Results are first presented where the wind conditions before the subjective event triggers were analyzed and compared with the overall crosswind intensity. Proposals of three new crosswind profiles are then formulated, based on the test data.

\section{Crosswind Correlation to High Speed Stability}

Crosswinds are often assumed to influence high speed stability performance, but there have also been discussions that issues with straight-line stability can occur regardless of wind conditions. This study shows that crosswinds were the dominant condition for causing stability issues, although the data show a few recordings of issues in still wind condition.

Table 3: The frequency of triggers in intervals on the crosswind change corresponding to levels on the Beaufort scale, compared to the complete data set.

\begin{tabular}{ccrrrr}
\hline & Gust conditions & & \multicolumn{2}{c}{ Percentage } \\
\hline Beaufort scale & Side wind change, $\Delta w_{y},[\mathrm{~m} / \mathrm{s}]$ & & Triggers & All data \\
\hline $0,1 \& 2$ & $0-3.3$ & & $16 \%$ & $42 \%$ \\
3 & $3.4-5.4$ & & $35 \%$ & $44 \%$ \\
4 & $5.5-7.9$ & & $71 \%$ & $12 \%$ \\
5 & $8.0-10.7$ & $1 \%$ & $2 \%$ \\
6 & $10.8-13.3$ & $100 \%$ & $100 \%$
\end{tabular}




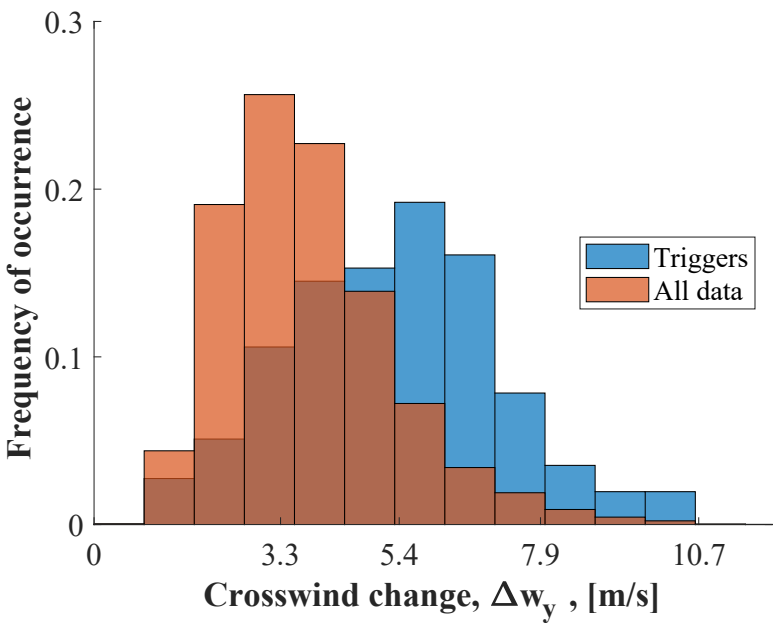

Figure 9: The distribution of the change in crosswind magnitude, for the trigger data and the complete data set.

The test data from the weeks of data acquisition showed that the vehicle was subjected to crosswind which mainly varied between 0 to 3 on the Beaufort wind scale, corresponding to wind changes between $0-$ $5.4 \mathrm{~m} / \mathrm{s}$, see All data in Table 3 The subjective evaluation of the test drivers resulted in 255 trigger events when substantial stability issues were experienced. The wind conditions were analyzed in the $3 \mathrm{~s}$ time interval before each trigger. It is evident from Table 3 that stronger crosswinds were more common at these trigger events, compared to the complete data set of the crosswind. The distribution of the data was also presented in Figure 9 where the dark brown color represents the overlapping data between the two data sets. The discrepancy between the wind conditions at the triggers and the complete data set indicates that changes in crosswind was an underlying factor for issues with high speed stability performance in this study. Half of all triggers occur in crosswinds with level 4 or above on the Beaufort scale, which only represents $14 \%$ of the total wind data. This statistically correlates a varying crosswind with decreased high speed stability performance. However, it should also be noted that $16 \%$ of the triggers occur in conditions with no or little wind $(0,1 \& 2$ on the Beaufort scale). It must, therefore, be assumed that driving stability issues might occur without any crosswind, even though these results show that the majority of the instabilities occur in changing crosswind conditions.

The resulting relative flow angle, $\psi$, is dependent on the vehicle speed, $v_{x}$, and the wind components, $w_{x}$ and $w_{y}$, as presented in Figure 1 and Equation 5 When driving at $140 \mathrm{~km} / \mathrm{h}$ without any head- or tailwind, a change in crosswind of $7 \mathrm{~m} / \mathrm{s}$ results in a relative flow angle change of $10 \mathrm{deg}$. Table 4 shows the frequency of occurrence of the flow angle change during the $3 \mathrm{~s}$ interval, at the triggers and for the complete data set. It is evident that Tables 3 and 4 display overlapping information. However, since the test procedure included different vehicle velocities

Table 4: The frequency of triggers in intervals on the flow angle change, compared to the complete data set.

\begin{tabular}{crr}
\hline & \multicolumn{2}{c}{ Percentage } \\
\cline { 2 - 3 } Flow angle change, $\Delta \psi,[\mathrm{deg}]$ & Triggers & All data \\
\hline $0-4$ & $18 \%$ & $36 \%$ \\
$4-6$ & $23 \%$ & $39 \%$ \\
$6-8$ & $34 \%$ & $16 \%$ \\
$8-10$ & $11 \%$ & $5 \%$ \\
$10-12$ & $9 \%$ & $3 \%$ \\
$>12$ & $5 \%$ & $1 \%$ \\
\hline & $100 \%$ & $100 \%$
\end{tabular}

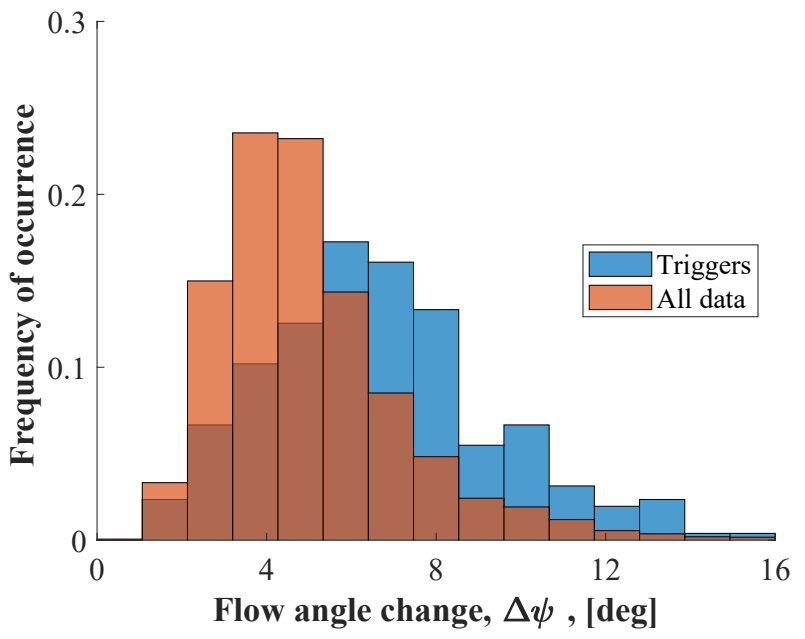

Figure 10: The distribution of the change in flow angle, for the trigger data and the complete data set.

the resulting flow angles could be of interest, at least for comparison with other studies. Only one-fourth of the complete data set had a varying flow angle above $6 \mathrm{deg}$, but $59 \%$ of the triggers were recorded at these flow conditions. Nevertheless, Table 4 indicates that gusts above $10 \mathrm{deg}$ were rare, but often causing stability issues. The distributions are presented in Figure 10 where the trigger data of the flow angle show a smaller discrepancy from the complete data set than the crosswind magnitude in Figure 9 The change in crosswind magnitude was, thus, the more relevant measure, of the two different lateral flow quantities.

Figure 11 presents the distribution for the change in headwind, $\Delta w_{x}$. Although the discrepancy between the trigger data and the complete data set was small, a change in magnitude above $5 \mathrm{~m} / \mathrm{s}$ showed an increased occurrence of subjective trigger events.

A frequent occurrence of zero-crossings in wind gusts has been seen in previous studies [29]. Likewise, by focusing on the triggers with gusty crosswind conditions (Beaufort scale $\geq 3$ ), it was found that the incoming flow angle changed sign and surpassed zero with at least $1 \mathrm{deg}$ in $83 \%$ of the triggers, during the $3 \mathrm{~s}$ interval before the trigger events, see Table 5 Moreover, according to [19] zero-crossing has been found to result in overshoots in forces and moments. This together

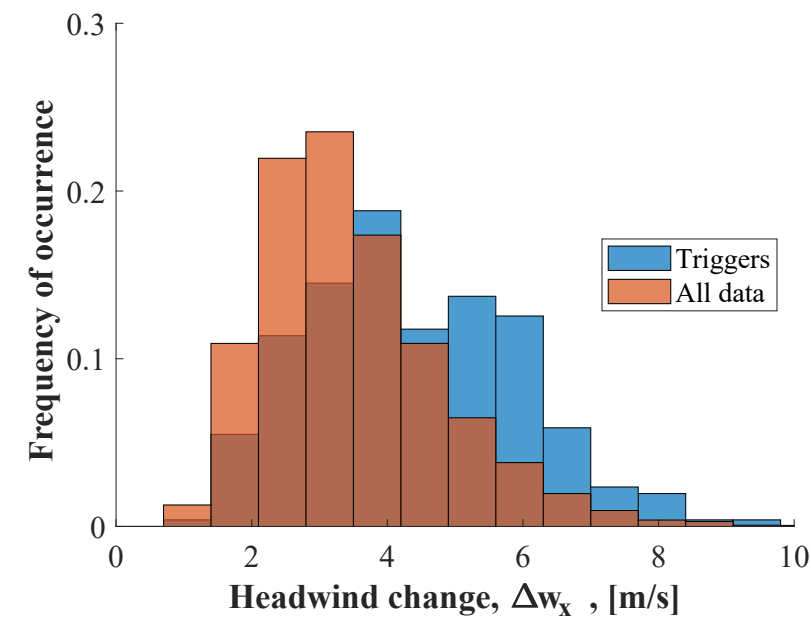

Figure 11: The distribution of the change in headwind magnitude, for the trigger data and the complete data set. 


\begin{tabular}{cc}
\hline \multicolumn{2}{c}{ zero-crossing } \\
No & Yes \\
\hline $17 \%$ & $83 \%$ \\
\hline
\end{tabular}

with the statistical evidence presented in Table 5 can thus demonstrate the importance of including a zero-crossing of the flow angle to fully evaluate high speed stability performance in gusty wind conditions. However, when analyzing the complete set of wind data above 3 on the Beaufort scale, the same ratio of zero-crossing gusts as the trigger data in Table 5 was found. No discrepancy between the trigger data and the complete data set implies that the zero-crossing does not increase the frequency of a subjective trigger. However, Table 5 still indicates that a realistic crosswind gust usually includes a zero-crossing.

\section{Crosswind Gust Profiles}

The natural wind is turbulent and highly stochastic, meaning that none of the crosswind gusts measured at the trigger events were identical. Nevertheless, certain patterns could be observed and a broad classification was done in terms of gust profiles. The profiles were defined mathematically, to enable adoption in numerical flow simulations and the opportunity to conduct studies with equal boundary conditions.

The piecewise function of crosswind gust, inspired by Favre and Efraimsson [24], can be seen in Equation 7] and Figure 12 The function has a parameter for the initial value of the crosswind, $w_{y}^{\text {start }}$, and an end value, $w_{y}^{\text {end }}$. Many of the on-road measurements indicated that these parameters should be set to zero, but there were also instances where the vehicle was subjected to a constant crosswind and a rapid change from that steady-state condition caused a subjective trigger event. The equation was therefore designed so that profiles could be created where the initial value of the crosswind could be chosen. The other two parameters of the crosswind magnitude were the maximum, $w_{y}^{\max }$, and the minimum, $w_{y}^{\text {min }}$, values. The crosswind magnitude starts to change from the initial starting value at the time $t_{0}$ and the parameter of the build-up time, $\Delta t_{b}$, determines how fast the maximum value is reached. The pausing time at the maximum and minimum crosswind magnitude

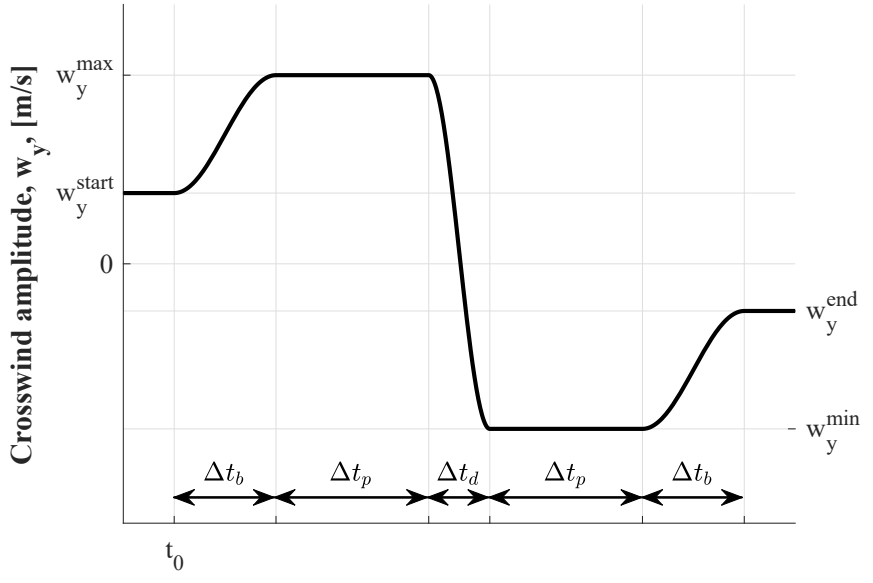

Time, t, $[\mathbf{s}]$

Figure 12: Example gust profile, built using Equation 7 with dimensions for the equation parameters of magnitude and time.

is set by the parameter $\Delta t_{p}$ and the time of the drop between the maximum and minimum values is determined by $\Delta t_{d}$. The pausing and build-up times are symmetrical for the first and second half of the gust.

An example gust, built from Equation 7. can be seen in Figure 12 The parameters give the function flexibility to represent many different crosswind gust profiles. The start, maximum, minimum and end values can freely be chosen between positive and negative numbers without breaking the function.

The crosswind gust function has been defined in the time domain. However, it could be of interest to describe the crosswind in space $\left(w_{y}(x)\right)$. This can be done by substituting time with distance and vehicle velocity, $t=x / v_{x}$, and similarly with the time durations, $\Delta t_{i}=$ $\Delta x_{i} / v_{x}$, in Equation 7 Moreover, to design a gust profile of desired vehicle lengths, $n L$, the distances can be set using $x=n L$ and in the time domain, $t=n L / v_{x}$.

The measured wind data in proximity to the subjective event triggers were classified into four profiles (A-D), and will be discussed next. All profiles were build using Equation 7

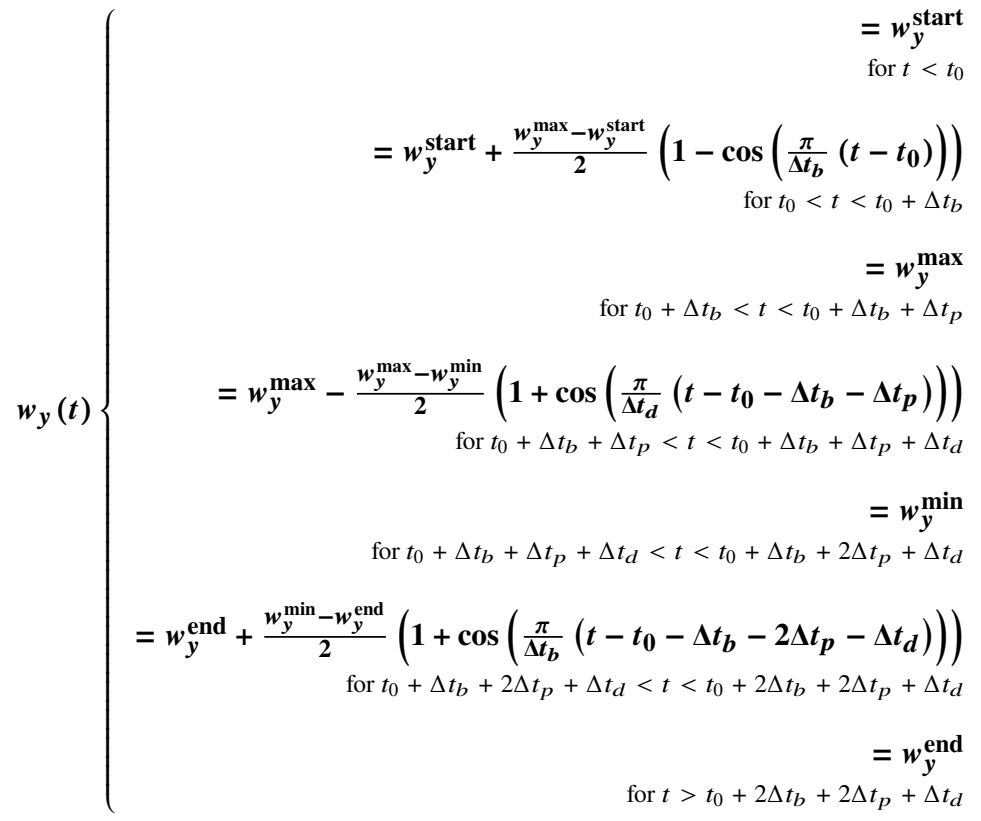




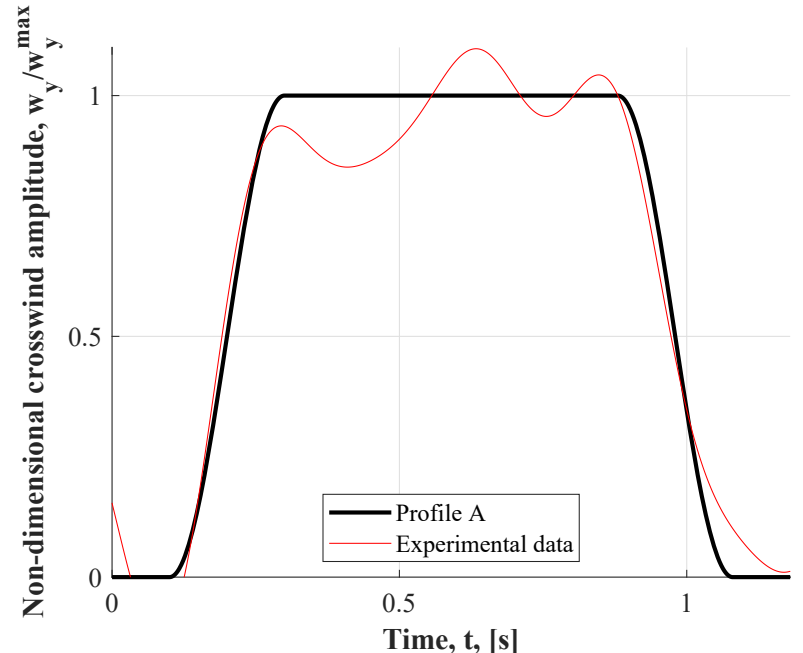

Figure 13: Profile A, using Equation 7 with $w_{y}^{\text {start }}=w_{y}^{\text {end }}=0, w_{y}^{\max }=w_{y}^{\min }=$ $5.00 \mathrm{~m} / \mathrm{s}, t_{0}=0.10 \mathrm{~s}, \Delta t_{b}=0.20 \mathrm{~s}, \Delta t_{p}=0.29 \mathrm{~s}$ and $\Delta t_{d}=0$. The profile is compared with experimental data.

\section{Profile A: ISO Standard}

Gust profile A is characterized by the quick ramp-up and ramp-down of the crosswind and a relatively long magnitude pause at the maximum crosswind magnitude without any zero-crossing, see Figure 13 The gust profile starts and ends with no crosswind and is the profile that best represents the crosswind sensitivity testing at crosswind facilities, described in ISO 12021:2010 [23]. This type of crosswind was however rare during the experimental on-road testing at the test track.

\section{Profile B: Sine Wave}

Gust profile B is characterized by a continuously changing crosswind, with a zero-crossing between two peak values, see Figure 14 This type of crosswind gusts was one of the most frequent in the experimental data, at the subjective trigger events. The regularly changing crosswind implies that the drop time is longer than the build-up time, $\Delta t_{d}>\Delta t_{b}$.

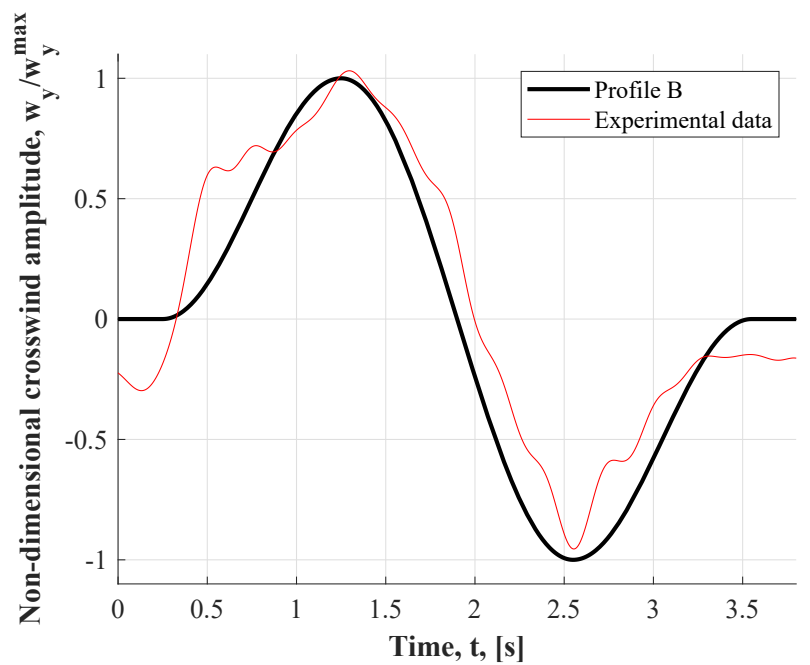

Figure 14: Profile B, using Equation 7 with $w_{y}^{\text {start }}=w_{y}^{\text {end }}=0, w_{y}^{\max }=$ $-w_{y}^{\min }=4.50 \mathrm{~m} / \mathrm{s}, t_{0}=0.25 \mathrm{~s}, \Delta t_{b}=1.00 \mathrm{~s}, \Delta t_{p}=0$ and $\Delta t_{d}=1.30 \mathrm{~s}$. The profile is compared with experimental data.

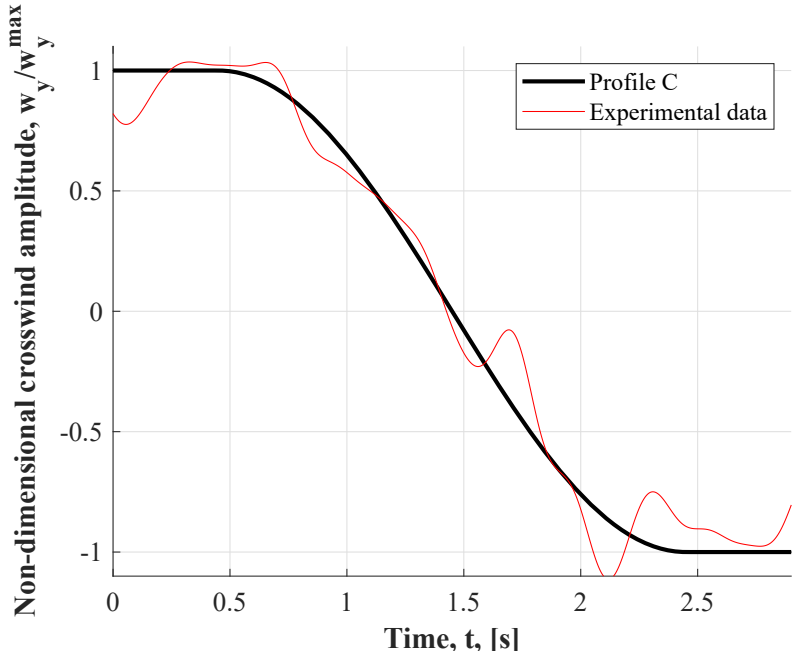

Figure 15: Profile C, using Equation 7 with $w_{y}^{\text {start }}=-w_{y}^{\text {end }}=w_{y}^{\max }=-w_{y}^{\min }=$ $3.50 \mathrm{~m} / \mathrm{s}, t_{0}=0.45 \mathrm{~s}, \Delta t_{b}=\Delta t_{p}=J_{\text {and }} \Delta t_{d}=2.00 \mathrm{~s}$. The profile is compared with experimental data.

\section{Profile C: Ramp/step}

Gust profile $\mathrm{C}$ is characterized by a simple transition between two levels in the magnitude of the crosswind. The example in Figure 15 includes a zero-crossing, but the experimental data also showed examples of a quick ramp down from a constant crosswind to no crosswind. The build-up and pause times are set to zero in this profile, $\Delta t_{b}=\Delta t_{p}=0$, and the initial crosswind magnitude equals the maximum magnitude, $w_{y}^{\text {start }}=w_{y}^{\max }$, and the end and minimum magnitudes are also equal, $w_{y}^{\min }=w_{y}^{\text {end }}$. A step function of the crosswind can be created by decreasing the drop time duration towards zero, $\Delta t_{d} \rightarrow 0$.

\section{Profile D: Build-up and Rapid Drop}

Gust profile D is characterized by a slow build-up time and a rapid drop including a zero-crossing, see Figure 16 This quick change, including a zero-crossing was noted to cause substantial stability issues. The profile is similar to profile $\mathrm{B}$, except that the drop time is shorter than the build-up time, $\Delta t_{d}<\Delta t_{b}$.

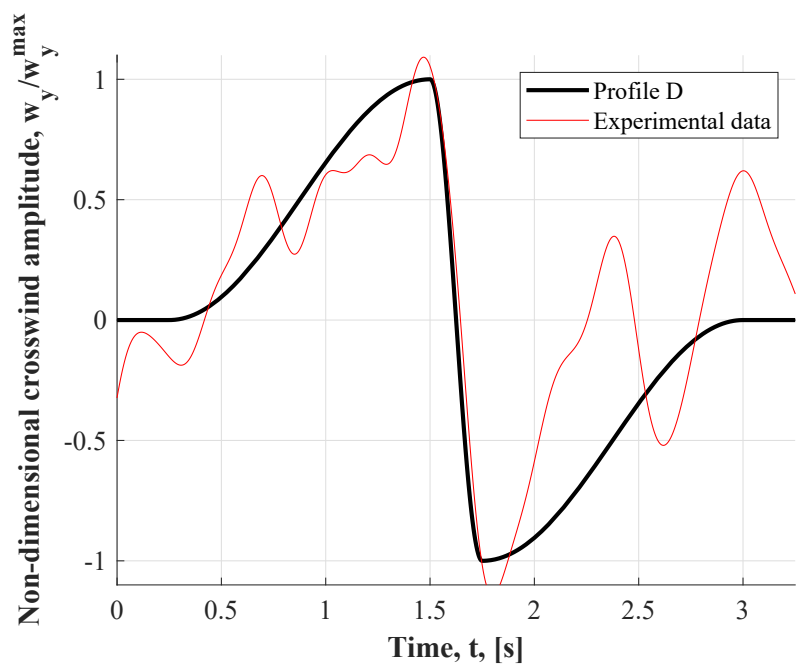

Figure 16: Profile B, using Equation 7 with $w_{y}^{\text {start }}=w_{y}^{\text {end }}=0, w_{y}^{\max }=$ $-w_{y}^{\min }=3.50 \mathrm{~m} / \mathrm{s}, t_{0}=0.25 \mathrm{~s}, \Delta t_{b}=1.25 \mathrm{~s}, \Delta t_{p}=0$ and $\Delta t_{d}=0.25 \mathrm{~s}$. The profile is compared with experimental data. 


\begin{tabular}{ccccc}
\hline \multicolumn{5}{c}{ Gust profile } \\
$\mathrm{A}$ & $\mathrm{B}$ & $\mathrm{C}$ & $\mathrm{D}$ & Other \\
\hline $6.0 \%$ & $22.7 \%$ & $23.1 \%$ & $13.0 \%$ & $35.2 \%$ \\
\hline
\end{tabular}

All gusts with crosswind variations above 3 on the Beaufort scale were visually inspected and sorted into this classification. Table 7 shows the frequency of occurrence for the gust profiles. The least common profile (A) was included because of use in previous studies and association with the ISO standard. Profile B and C occurred most frequently in the test data. The fourth profile (D) did not occur as frequently. However, trigger events with these types of slow-to-rapidly changing wind conditions were often particularly noted by the drivers to have stability issues. Profiles $\mathrm{C}$ and $\mathrm{D}$ can be seen as variants of a common base profile and that their combined ratio would be $36.1 \%$. However, it is evident that the wind data were highly irregular since $35.2 \%$ of the crosswind gusts did not fit into any of the four profiles. Nevertheless, this classification of gust profiles enhances the possibility to use real-world inspired crosswind gust profiles in virtual simulations.

\section{Quantitative Assessment}

Formulating quantitative objective measures for subjective evaluation often proves to be a difficult task. In this study, a statistical correlation using the subjective event triggers provided by the drivers at instances of substantial issues with high speed stability was implemented. The correlation focuses on discrepancies between measured data close to the trigger events and the complete data set to indicate the exceptional vehicle motion responses experienced by the drivers right before they pressed the trigger button.

The statistical correlation is followed by an attempt to use a methodology for predicting the locations of the subjective triggers based on the objective data, using the predictive filter. The filter setup provides information about quantitative measures to be used to assess high speed stability performance.

\section{Vehicle Motion Correlation to High Speed Stability}

The driver constantly gathers information from the human senses when driving. The motion of the vehicle acts on the driver via the seat and the steering wheel provides certain feedback. Sight is vital during any driving scenario and hearing gives information on wind and weather, road surface and vehicle speed. All these senses are combined in the

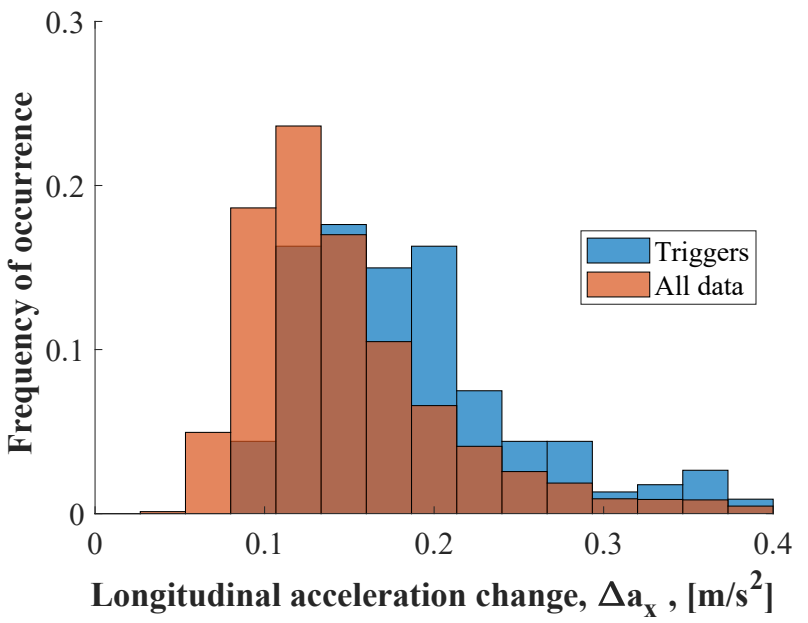

Figure 17: The distribution of the change in longitudinal acceleration, for the trigger data and the complete data set.

subjective assessment of high speed stability. This section focuses on the correlation between the vehicle motion response and the subjective evaluation, using the trigger events recorded by the drivers.

The six degrees of freedom (DoF) were measured during the testing, three accelerations in the local coordinate system of the vehicle and three angular velocities around the coordinate axes. The driver's lower back was used as the reference point for the accelerations, to capture the motion of the vehicle body experienced by the drivers. A time interval of $3 \mathrm{~s}$ before each subjective event trigger was classified as the region of instability, in similarity with the wind analysis in the previous section. The maximum magnitude was measured for the six signals in each of the triggers' regions of instability. These values are placed in intervals that can be seen in Table 6 The percentage of values at the intervals for the triggers, can be compared to the values of the complete data set, see All data in Table 6 Large discrepancies between the trigger data and the complete data set indicate that those vehicle motion conditions were extraordinary for the trigger events.

When analyzing the change in longitudinal acceleration, $\Delta a_{x}$, during the $3 \mathrm{~s}$ window, it is evident that the spread was low with $60 \%$ of all data in the interval of $0.1-0.2 \mathrm{~m} / \mathrm{s}^{2}$, see Table 6 . This was also the case for the trigger data. However, the table shows a small discrepancy between trigger data and the complete data set for the longitudinal acceleration. Figure 17 presents the distribution, where the small discrepancy can be seen.

In general, the change in lateral acceleration, $\Delta a_{y}$, proved to be greater

Table 6: The percentage of triggers in intervals for the vehicle's 6 DoF, compared to the complete data set.

\begin{tabular}{|c|c|c|c|c|c|c|c|c|c|c|c|c|c|}
\hline \multirow[b]{2}{*}{ Interval } & \multicolumn{2}{|c|}{$\Delta a_{x}\left[\mathrm{~m} / \mathrm{s}^{2}\right]$} & \multicolumn{2}{|c|}{$\Delta a_{y}\left[\mathrm{~m} / \mathrm{s}^{2}\right]$} & \multicolumn{2}{|c|}{$\Delta \omega_{z}[\mathrm{deg} / \mathrm{s}]$} & \multirow[b]{2}{*}{ Interval } & \multicolumn{2}{|c|}{$\Delta \omega_{x}[\mathrm{deg} / \mathrm{s}]$} & \multicolumn{2}{|c|}{$\Delta \omega_{y}[\mathrm{deg} / \mathrm{s}]$} & \multicolumn{2}{|c|}{$\Delta a_{z}\left[\mathrm{~m} / \mathrm{s}^{2}\right]$} \\
\hline & Triggers & All data & Triggers & All data & Triggers & All data & & Triggers & All data & Triggers & All data & Triggers & All data \\
\hline $0-0.1$ & $3 \%$ & $18 \%$ & $0 \%$ & $0 \%$ & $0 \%$ & $0 \%$ & $0-0.3$ & $0 \%$ & $0 \%$ & $0 \%$ & $0 \%$ & $0 \%$ & $0 \%$ \\
\hline $0.1-0.2$ & $60 \%$ & $60 \%$ & $0 \%$ & $1 \%$ & $0 \%$ & $0 \%$ & $0.3-0.6$ & $0 \%$ & $0 \%$ & $1 \%$ & $0 \%$ & $25 \%$ & $24 \%$ \\
\hline $0.2-0.3$ & $23 \%$ & $11 \%$ & $1 \%$ & $11 \%$ & $0 \%$ & $1 \%$ & $0.6-0.9$ & $0 \%$ & $1 \%$ & $8 \%$ & $7 \%$ & $29 \%$ & $23 \%$ \\
\hline $0.3-0.4$ & $6 \%$ & $3 \%$ & $5 \%$ & $26 \%$ & $1 \%$ & $6 \%$ & $0.9-1.2$ & $2 \%$ & $4 \%$ & $17 \%$ & $15 \%$ & $15 \%$ & $14 \%$ \\
\hline $0.4-0.5$ & $2 \%$ & $1 \%$ & $19 \%$ & $26 \%$ & $2 \%$ & $15 \%$ & $1.2-1.5$ & $18 \%$ & $18 \%$ & $19 \%$ & $15 \%$ & $9 \%$ & $12 \%$ \\
\hline $0.5-0.6$ & $1 \%$ & $1 \%$ & $26 \%$ & $17 \%$ & $7 \%$ & $21 \%$ & $1.5-1.8$ & $24 \%$ & $22 \%$ & $14 \%$ & $12 \%$ & $6 \%$ & $9 \%$ \\
\hline $0.6-0.7$ & $2 \%$ & $1 \%$ & $18 \%$ & $8 \%$ & $12 \%$ & $19 \%$ & $1.8-2.1$ & $16 \%$ & $16 \%$ & $11 \%$ & $12 \%$ & $6 \%$ & $7 \%$ \\
\hline $0.7-0.8$ & $0 \%$ & $1 \%$ & $14 \%$ & $4 \%$ & $15 \%$ & $15 \%$ & $2.1-2.4$ & $13 \%$ & $16 \%$ & $11 \%$ & $12 \%$ & $3 \%$ & $5 \%$ \\
\hline $0.8-0.9$ & $1 \%$ & $1 \%$ & $7 \%$ & $1 \%$ & $19 \%$ & $10 \%$ & $2.4-2.7$ & $10 \%$ & $12 \%$ & $8 \%$ & $12 \%$ & $4 \%$ & $1 \%$ \\
\hline $0.9-1.0$ & $0 \%$ & $1 \%$ & $4 \%$ & $1 \%$ & $11 \%$ & $5 \%$ & $2.7-3.0$ & $9 \%$ & $7 \%$ & $7 \%$ & $9 \%$ & $1 \%$ & $1 \%$ \\
\hline$>1.0$ & $2 \%$ & $2 \%$ & $6 \%$ & $5 \%$ & $33 \%$ & $8 \%$ & $>3.0$ & $8 \%$ & $4 \%$ & $4 \%$ & $6 \%$ & $2 \%$ & $4 \%$ \\
\hline & $100 \%$ & $100 \%$ & $100 \%$ & $100 \%$ & $100 \%$ & $100 \%$ & & $100 \%$ & $100 \%$ & $100 \%$ & $100 \%$ & $100 \%$ & $100 \%$ \\
\hline
\end{tabular}




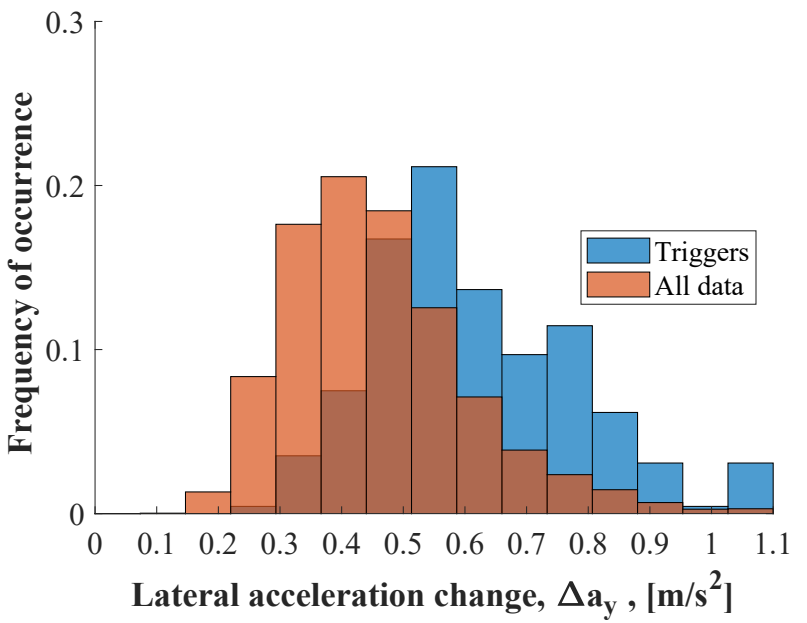

Figure 18: The distribution of the change in lateral acceleration, for the trigger data and the complete data set.

compared to the longitudinal acceleration. Hence, the driver is subjected to higher variations in lateral acceleration at normal straight-line driving. Also, the discrepancy between trigger data and the complete data set was greater for the lateral acceleration, indicating that this vehicle motion can be correlated to high speed stability performance, see Figure 18 For example, only $36 \%$ of the complete data magnitude variations above $0.5 \mathrm{~m} / \mathrm{s}^{2}$ while the number was $75 \%$ for the data at the trigger events.

The yaw velocity, $\omega_{z}$, and lateral acceleration, $a_{y}$, are motions in the road plane and they have a high correlation between themselves. Table 6 and Figure 19 show that there was an even larger discrepancy between triggers and all data for the change in yaw velocity, $\Delta \omega_{z}$, where $33 \%$ of the trigger data varies $>1.0 \mathrm{deg} / \mathrm{s}$ (compared to only $8 \%$ of the complete data set). Hence, high changes in lateral acceleration and yaw velocity both seem to correlate with low high speed stability performance. These vehicle motion responses can thus be used to formulate quantitative measures for high speed stability.

The roll velocity, $\omega_{x}$, pitch velocity, $\omega_{y}$, and vertical acceleration, $a_{z}$, had higher variations at normal straight-line driving compared to yaw velocity, longitudinal and lateral acceleration. Larger intervals were

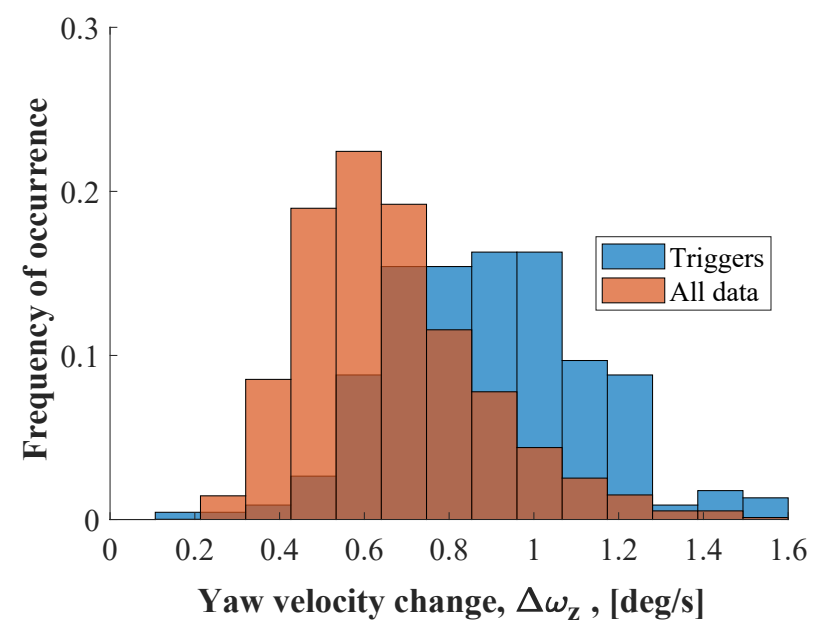

Figure 19: The distribution of the change in yaw velocity, for the trigger data and the complete data set.

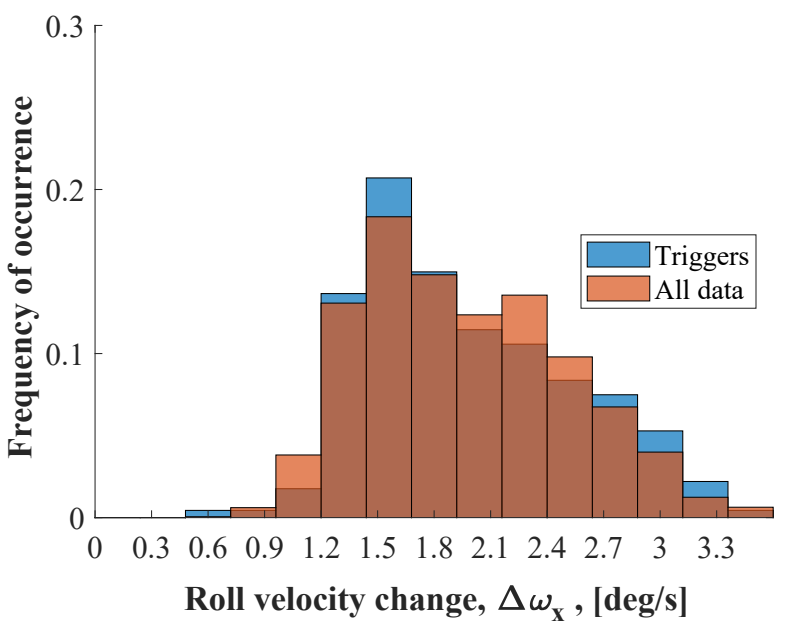

Figure 20: The distribution of the change in roll velocity, for the trigger data and the complete data set.

therefore used in Table 6 to capture their spread. According to Figure 20 the change in roll velocity, $\Delta \omega_{x}$, had almost no discrepancy between trigger data and the complete data set. Hence, it could be concluded, using this analysis method, that large changes in roll velocity were not the cause for the drivers' subjective triggers.

Similarly, no discrepancies could be seen for either the change in pitch velocity, $\Delta \omega_{y}$, or vertical acceleration, $\Delta a_{z}$, see Figures 21 and 22 . indicating that these were two other vehicle motions that were not related to the drivers' subjective assessment of substantial stability issues. So, even though the roll velocity, pitch velocity, and vertical acceleration generally had higher magnitude variations compared to the other three vehicle motions, they did not correlate with poor high speed stability performance. The oscillating and vibrating motions from the road are expected by the driver and were thus not evaluated as something exceptional.

\section{Prediction of Event Triggers}

The predictive filter, using the amplitude and duration of signal peakto-peak values to predict regions of stability issues, could not find

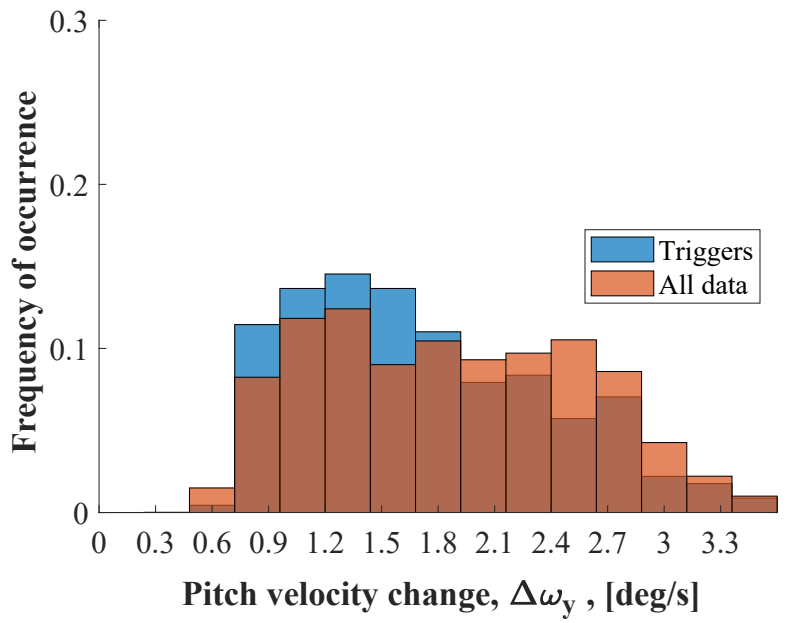

Figure 21: The distribution of the change in pitch velocity, for the trigger data and the complete data set. 


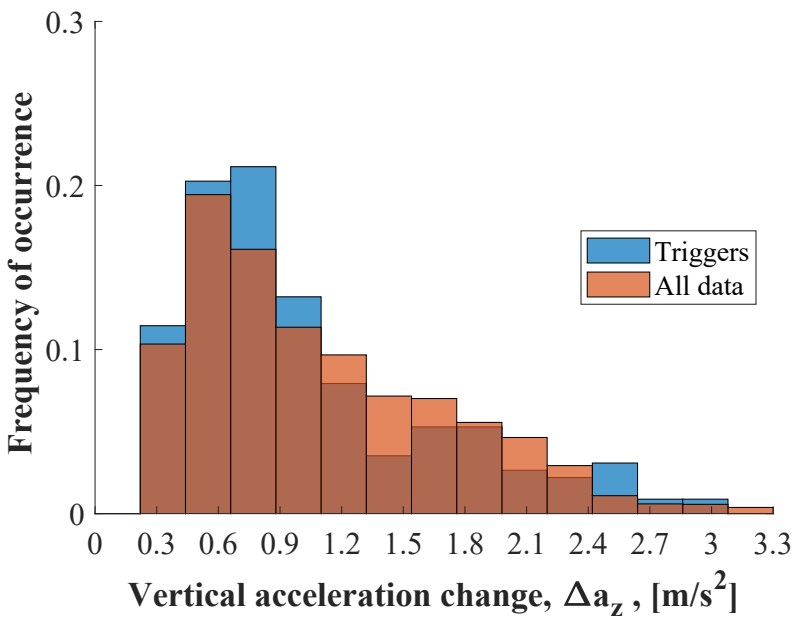

Figure 22: The distribution of the change in vertical acceleration, for the trigger data and the complete data set.

any good prediction for longitudinal and vertical acceleration, roll and pitch velocity, in similarity with the results presented above. The optimization strategy was designed to minimize the loss function, $f_{L}$, (Equation 6). The loss function was based on the confusion matrices produced by the prediction filter. The optimization was done on the two filter input parameters: signal peak amplitude and peak duration. The best (lowest) results of the loss function is presented in Table 8 where it is evident that the prediction filter performs best on the lateral acceleration and yaw velocity signals.

The confusion matrices for lateral acceleration and yaw velocity can be seen in Tables 9 and 10 respectively. The filter was able to predict the majority of the real trigger events, for both signals. However, the tables also show that most of the predicted triggers were false positives. It was expected that the filter would predict many false triggers due to the uncertainty of the test drivers' subjective assessment and noise in the test track data. Nevertheless, the fact that lateral acceleration and yaw velocity were the only two motions that could predict the majority of the real triggers without having thousands of false positive predictions again indicate that they show correlation with the subjective assessment. Furthermore, the prediction filter for these signals gave useful information on quantitative objective measures for high speed stability:

- A change in lateral acceleration higher than $\Delta a_{y}>0.58 \mathrm{~m} / \mathrm{s}^{2}$, within a time interval of $\Delta t=2.85 \mathrm{~s}$ was the solution found using optimization which resulted in the prediction accuracy presented in Table 9

- A change in yaw velocity higher than $\Delta \omega_{z}>0.84 \mathrm{deg} / \mathrm{s}$, within a time interval of $\Delta t=2.68 \mathrm{~s}$ was the solution found using optimization which resulted in the prediction accuracy presented in Table 10

Table 8: Loss function, $f_{L}$, results.

\begin{tabular}{lll}
\hline & & $f_{L}$ \\
\hline Longitudinal acceleration, & $a_{x}$ & 77.75 \\
Lateral acceleration, & $a_{y}$ & -7.50 \\
Vertical acceleration, & $a_{z}$ & 201.75 \\
Roll velocity, & $\omega_{x}$ & 206.50 \\
Pitch velocity, & $\omega_{y}$ & 202.00 \\
Yaw velocity, & $\omega_{z}$ & -33.00 \\
\hline
\end{tabular}

Table 9: Confusion matrix of the prediction filter outcome, for the lateral acceleration signal.

\begin{tabular}{crcc} 
& & \multicolumn{2}{c}{ Predicted } \\
\cline { 3 - 4 } & & Trigger & No trigger \\
\cline { 3 - 4 }$\cong$ & Trigger & 144 & 59 \\
& No trigger & 598 &
\end{tabular}

Table 10: Confusion matrix of the prediction filter outcome, for the yaw velocity signal

\begin{tabular}{|c|c|c|}
\hline & \multicolumn{2}{|c|}{ Predicted } \\
\hline & Trigger & No trigger \\
\hline $\begin{array}{r}\text { Trigger } \\
\text { No trigger }\end{array}$ & $\begin{array}{l}150 \\
556\end{array}$ & 53 \\
\hline
\end{tabular}

\section{Conclusions}

The purpose of this study has been to define relevant wind conditions and to find an objective methodology to evaluate high speed stability. Due to the subjective nature of the study along with the repeatability of the test track environment, it was difficult to quantify the total measurement uncertainties accurately. However, based on the extensive data set, the following conclusions could be drawn:

- The majority of the issues related to high speed stability occurred in gusty wind conditions. Hence, the analysis of high speed stability should include a realistic varying crosswind. The magnitude of the fluctuating crosswind component should exceed $5 \mathrm{~m} / \mathrm{s}$.

- A zero-crossing of the flow angle was seen in $83 \%$ of the crosswind gusts above 3 on the Beaufort scale. Although the zerocrossing did not increase the frequency of subjective trigger events, it was concluded that this property should be included in flow simulations to represent realistic wind conditions.

- A mathematical function of the crosswind gust profile was formulated and used to classify the experimental data into four wind profiles. The profiles showed a close representation of the data and can be used as boundary conditions in virtual simulations.

- The lateral acceleration and the yaw velocity were the only two vehicle motions that showed a statistical correlation between high variations and an increased frequency of stability issues. The change in longitudinal acceleration had a small yet uncertain correlation, and the roll velocity, pitch velocity, and vertical acceleration showed no correlation between the magnitude variations and stability issues.

- The predictive filter could predict the majority of the real triggers using the lateral acceleration and yaw velocity signals. However, the predictions resulted in more false positive than true positive triggers. Some of the false triggers could be a result of the difference in subjective evaluation between drivers and days of driving, and the uncertainty of the test track data. Nevertheless, it must be concluded that the filter does not capture the complete subjective assessment objectively, but that it can be used as a guiding tool to virtually assess high speed stability.

- The optimization of the prediction filter input parameters resulted in a solution where a trigger could best be predicted if the change in lateral acceleration was higher than $\Delta a_{y}>0.58 \mathrm{~m} / \mathrm{s}^{2}$, within a time interval of $\Delta t=2.85 \mathrm{~s}$, and similarly if the yaw velocity was higher than $\Delta \omega_{z}>0.84 \mathrm{deg} / \mathrm{s}$, within a time interval of $\Delta t=$ $2.68 \mathrm{~s}$. Hence, these values can be used as objective measures to quantitatively assess high speed stability. 


\section{References}

[1] Baker, C. J. and Reynolds, S. "Wind-induced accidents of road vehicles". In: Accident Analysis \& Prevention 24.6 (1992), pp. 559-575. DOr: 10.1016/0001-4575(92)90009-8

[2] Kumar, A., Sebben, S., Sällström, E., Jacobson, B. J. H., and Broniewicz, A. "Analysis of Subjective Qualitative Judgement of Passenger Vehicle High Speed Drivability due to Aerodynamics". In: Energies 12.14 (2019). DOI: 10.3390/en12142839.

[3] Howell, J. and Panigrahi, S. "Aerodynamic Side Forces on Passenger Cars at Yaw". Conference Paper. 2016. Dor: 10.4271/ 2016-01-1620

[4] Hucho, W.-H. "Aerodynamics of Road Vehicles". Fourth edition. SAE International, 1998.

[5] MacAdam, C. C., Sayers, M. W., Pointer, J. D., and Gleason, M. "Crosswind Sensitivity of Passenger Cars and the Influence of Chassis and Aerodynamic Properties on Driver Preferences". In: Vehicle System Dynamics 19.4 (1990), pp. 201-236. DoI: 10.1080/00423119008968942

[6] Barth, R. "Effect of Unsymmetrical Wind Incidence on Aerodynamic Forces Acting on Vehicle Models and Similar Bodies". Conference Paper. 1965. Dor: https://doi .org/10 . 4271/ 650136

[7] Milliken, W. F., Dell'Amico, F., and Rice, R. S. "The Static Directional Stability and Control of the Automobile". Conference Paper. 1976. Dor: $10.4271 / 760712$

[8] Buchheim, R., Maretzke, J., and Piatek, R. "The Control of Aerodynamic Parameters Influencing Vehicle Dynamics". In: SAE Paper (1985), pp. 850279-850279. Dor: 10.4271/850279

[9] Howell, J. and Le Good, G. "The Influence of Aerodynamic Lift on High Speed Stability". In: SAE Technical Paper SeriesTechnial 01.0651 (1999), pp. 8-8. Dor: 10.4271/1999-01-0651

[10] Windsor, S. and Le Good, G. "The Influence of Aerodynamic Lift on High Speed Stability". In: Autotech 93. Vol. 01, pp. 8-8.

[11] Sims-Williams, D. "Cross Winds and Transients: Reality, Simulation and Effects". In: SAE International Journal of Passenger Cars - Mechanical Systems 4.1 (2011), pp. 172-183. DoI: 10.4271/2011-01-0172

[12] Chadwick, A., Garry, K., and Howell, J. "Transient Aerodynamic Characteristics of Simple Vehicle Shapes by the Measurement of Surface Pressures". In: SAE Technical Paper Series (2000). DOI: https://doi.org/10.4271/2000-01-0876

[13] Hucho, W. H. and Emmelmann, H. J. "Theoretical Prediction of the Aerodynamic Derivatives of a Vehicle in Cross Wind Gusts". In: SAE Technical Paper Series 730232 (1973), pp. 892900. DOI: $10.4271 / 730232$

[14] Cooper, K. R. and Watkins, S. "The Unsteady Wind Environment of Road Vehicles, Part One: A Review of the On-road Turbulent Wind Environment". In: SAE Technical Paper Series. DoI: 10 . 4271/2007-01-1236

[15] Wordley, S. and Saunders, J. W. "On-road Turbulence". In: SAE Int. J. Passeng. Cars - Mech. Syst. 1.1 (2008), pp. 341-360. DoI: https://doi.org/10.4271/2008-01-0475

[16] Wordley, S. and Saunders, J. W. "On-road Turbulence: Part 2". In: SAE International Journal of Passenger Cars - Mechanical Systems 2.1 (2009), pp. 111-137. DOI: 10 . 4271/2009-010002 .

[17] Watkins, S. and Cooper, K. R. "The Unsteady Wind Environment of Road Vehicles, Part Two: Effects on Vehicle Development and Simulation of Turbulence". In: SAE Technical Paper Series. DoI: 10.4271/2007-01-1237
[18] Wagner, A. and Wiedemann, J. "Crosswind Behavior in the Driver 's Perspective". In: SAE Technical Paper Series 724 (2002). DOI: $10.4271 / 2002-01-0086$

[19] Theissen, P. "Unsteady Vehicle Aerodynamics in Gusty Crosswind". PhD thesis. Technical University of Munich, 2012.

[20] Fukagawa, T., Shimokawa, S., Itakura, E., Nakatani, H., and Kitahama, K. "Modeling of Transient Aerodynamic Forces based on Crosswind Test". In: SAE Int. J. Passeng. Cars - Mech. Syst. 9.2 (2016), pp. 572-582. Dor: https : //doi .org/10 .4271/ 2016-01-1577

[21] Nakasato, K. et al. "Coupled 6DoF Motion and Aerodynamic Crosswind Simulation Incorporating Driver Model". In: $S A E$ Int. J. Passeng. Cars - Mech. Syst. 10.2 (2017), pp. 662-670. DoI: https://doi.org/10.4271/2017-01-1525

[22] Lewington, N., Ohra-aho, L., Lange, O., and Rudnik, K. "The Application of a One-Way Coupled Aerodynamic and MultiBody Dynamics Simulation Process to Predict Vehicle Response during a Severe Crosswind Event". Conference Paper. 2017. Dor: https://doi.org/10.4271/2017-01-1515

[23] "ISO 12021:2010 Road vehicles - Sensitivity to lateral wind - Open-loop test method using wind generator input". International Organization for Standardization, 2010.

[24] Favre, T. and Efraimsson, G. "An Assessment of Detached-Eddy Simulations of Unsteady Crosswind Aerodynamics of Road Vehicles". In: Flow, Turbulence and Combustion 87.1 (2011), pp. 133-163. DOI: 10.1007/s10494-011-9333-4

[25] Favre, T. "Aerodynamics simulations of ground vehicles in unsteady crosswind". PhD thesis. Royal University of Technology, 2011.

[26] Forbes, D. C., Page, G. J., Passmore, M. A., and Gaylard, A. P. "A Fully Coupled, 6 Degree-of-Freedom, Aerodynamic and Vehicle Handling Crosswind Simulation using the DrivAer Model". In: SAE International Journal of Passenger Cars - Mechanical Systems 9.2 (2016). Dor: $10.4271 / 2016-01-1601$.

[27] Carbonne, L., Winkler, N., and Efraimsson, G. "Use of Full Coupling of Aerodynamics and Vehicle Dynamics for Numerical Simulation of the Crosswind Stability of Ground Vehicles". In: SAE International Journal of Commercial Vehicles 9.2 (2016), pp. 359-370. DOI: 10.4271/2016-01-8148

[28] Huang, T., Li, S., Wan, Z., and Gu, Z. "Investigation of vehicle stability under crosswind conditions based on coupling methods". In: Proceedings of the Institution of Mechanical Engineers, Part D: Journal of Automobile Engineering (2019). DOI: 10.1177/0954407018822424

[29] Wojciak, J. "Quantitative Analysis of Vehicle Aerodynamics during Crosswind Gusts". PhD thesis. Technical University of Munich, 2012.

[30] Chandrasekaran, K., Rao, N., Palraj, S., Kurella, C., and Lebbai, M. n. "Objective Drivability Evaluation on Compact SUV and Comparison with Subjective Drivability". Conference Paper. 2017. DoI: $10.4271 / 2017-26-0153$

[31] Willumeit, H. P., Müller, K., Dödlbacher, G., and Matheis, A. "Method to Correlate Vehicular Behaviour and Driver's Judgement under Side Wind Disturbances". In: Vehicle System Dynamics 17 (1988), pp. 508-524. Dor: 10.1080/ Q0423118808969292

[32] Aeroprobe Corporation. "Standard Probe User Manual, Document No. 90001-02-UMN-02". 2015.

[33] Schuetz, T. "Aerodynamics of Road Vehicles". Fifth Edition. 2015. ISBN: 978-0-7680-7977-7. DoI: $10.4271 / \mathrm{r}-430$ 
[34] Dewesoft GmbH. "DS-IMU/Gyro User Manual". 2013.

[35] Feldt, R. "BlackBoxOptim.jl". https : / / github . com / robertfeldt/BlackBox0ptim.jl 2018.

\section{Contact Information}

\section{Adam Brandt}

Dept. of Mechanics and Maritime Sciences

Chalmers University of Technology

41296 Gothenburg, Sweden

adam.brandt@chalmers.se 